\title{
MiR-133a-3p transferred by circulating microvesicles derived from myocardial ischemic preconditioning protects cardiomyocytes against hypoxia/reoxygenation injury
}

\section{Junyu Zhao}

Department of Pharmacology, School of Basic Medical Sciences, Tianjin Medical University

\section{Qian Zhu}

Department of Pharmacology, School of Basic Medical Sciences, Tianjin Medical University

\section{Man Shang}

Department of Pharmacology, School of Basic Medical Sciences, Tianjin Medical University

Miao Liu

Human Resource Department, Tianjin University of Traditional Chinese Medicine

\section{Yilu Wang}

Department of Pharmacy, Tianjin Medical University General Hospital

\section{Yeyi Li}

Department of Pharmacology, School of Basic Medical Sciences, Tianjin Medical University

\section{Yanna Wu}

Department of Pharmacology, School of Basic Medical Sciences, Tianjin Medical University

\section{Yanxia Liu}

Department of Pharmacology, School of Basic Medical Sciences, Tianjin Medical University

Junqiu Song ( $\nabla$ songjunqiu@tmu.edu.cn )

https://orcid.org/0000-0001-8152-7952

\section{Research article}

Keywords: MiR-133a-3p, Microvesicles, Ischemic preconditioning, Hypoxia/reoxygenation injury, Cardioprotection, Endoplasmic reticulum stress

Posted Date: February 28th, 2020

DOI: https://doi.org/10.21203/rs.2.24805/v1

License: (c) (i) This work is licensed under a Creative Commons Attribution 4.0 International License. Read Full License 


\section{Abstract}

Background Microvesicles (MVs) are submicron membrane vesicles as mediators of intercellular communication. The aim of our study was to investigate protective mechanism of circulating MVs derived from ischemic preconditioning (IPC-MVs) on myocardial I/R injury.

Results Administration of IPC-MVs reduced infarct size and activity of lactate dehydrogenase (LDH) in myocardial I/R injury in vivo. Meanwhile, IPC-MVs could increase cell viability and reduce LDH activity in hypoxia/reoxygenation (H/R) injured H9c2 cells in vitro. Microarray analysis demonstrated that miR133a-3p expression in IPC-MVs increased apparently compared with Sham-MVs. We found that miR133a-3p increased cell viability, decreased LDH activity and apoptosis, as well as suppressed H/Rinduced endoplasmic reticulum stress (ERS). MVs induced by hypoxic preconditioning enriched with FAM-miR-133a-3p allowed the transfer of miR-133a-3p to target cells. In addition, miR-133a-3p was significantly increased in $\mathrm{H} / \mathrm{R}$ injured $\mathrm{H} 9 \mathrm{c} 2$ cells by treatment with IPC-MVs. Epidermal growth factor receptor (EGFR) is a target gene of miR-133a-3p. AG1478 (EGFR inhibitor) significantly increased cell viability, decreased LDH activity and ERS-induced apoptosis in H9c2 cells under H/R injury.

Conclusions The findings of this study showed that IPC-MVs exerted cardioprotective effects by transferring miR-133a-3p into H/R injured cardiomyocytes targeting EGFR, thus attenuating ERS-induced apoptosis. MiR-133a-3p transferred by IPC-MVs may provide a novel therapy for myocardial I/R injury.

\section{Background}

Ischemic heart disease (IHD) is a leading cause of lethal cardiovascular disease with high morbidity and mortality worldwide. Giving blood reperfusion and oxygen supply to ischemic myocardium, at the same time, aggravated myocardial injury, that is called ischemia/reperfusion (I/R) injury[1]. It is well-known that ischemic preconditioning (IPC) is one of the most powerful cardioprotective interventions to reduce myocardial I/R injury[2], which could induce extracellular vesicles (EVs) such as microvesicles (MVs) and exosomes release and changes in microRNA (miRNA) expression in cardiomyocytes[3, 4], but the underlying mechanism remains elusive. Recently, it has been shown in our study that circulating MVs released from the heart after IPC (IPC-MVs) are required for cardioprotection by IPC[5] and that IPC-MVs could protect against myocardial I/R injury by regulating GRP78 and CHOP expression in endoplasmic reticulum pathway[6]. However, elucidating the protective mechanisms of IPC-MVs needs further molecular experimentation.

MVs are submicron membrane vesicles with the size range of $100 \sim 1000 \mathrm{~nm}$ in diameter shed from almost all eukaryotic cells during activation or apoptosis[7]. Multiple experiments have shown that MVs derived from various cell types could play intercellular communication role in diagnostic and therapeutic effects of cardiovascular diseases, mainly by delivering proteins, cytokines and gene messages such as mRNAs and microRNAs (miRNAs) to target cells[8]. Among the components of MVs, miRNAs have been shown to play important roles that regulate the pathophysiological consequences of myocardial I/R 
injury[9], which can be released from various cells by MVs that protect them from degradation and guarantee their stability and circulation through the blood stream[10], suggesting miRNA in MVs could be a potent therapeutic target and tool for protecting myocardium against I/R injury.

MiRNAs are a class of highly conserved endogenous, short (18 22 nucleotides) non-coding RNA molecules, which regulate gene expression post-transcriptionally[11]. MiRNAs negatively impact the expression of their target mRNAs mainly through interaction with the complementary sequences in the $3^{\prime}$ untranslated region ( $3^{\prime} \mathrm{UTR}$ ) and are well-known to play important roles in mediating intracellular signaling, trafficking and cell therapy in the cardiovascular system[12].

Intercellular communication is generally achieved through direct cell-to-cell contact or transfer of secreted paracrine molecules. Given as biological vectors, the interactions of released MVs with target cells, which relay specific biological information, is considered as an effective form of cellular communication.

Recent studies have shown that circulating MVs play an important role in myocardial ischemic injury through carrying well-defined considerable miRNAs and that MVs-mediated transfer of miRNAs between cardiomyocytes contributes to the rescuing of myocardial I/R injury[13]. However, limited information is available regarding the potential biological functions of miRNAs from IPC-MVs. Here, we investigated the protective effects of IPC-MVs in myocardial I/R injury model in vivo and H/R injury model in vitro, then using microarray and qRT-PCR analysis, we explored the differential expression of miRNAs in circulating MVs derived from the rats subjected to IPC or sham operation. We observed that miR-133a-3p expression in the IPC-MVs was significantly higher than that in the Sham-MVs. Previous studies have demonstrated that miR-133a-3p plays a cardioprotective role in myocardial I/R injury by downregulating the expression of the pro-apoptotic proteins DAPK2[14] and TAGLN2[15] and, that miR-133a-3p promotes the cardiogenic differentiation of human mesenchymal stem cells (hMSCs) by targeting epidermal growth factor receptor (EGFR)[16].

Based on the above studies, we hypothesized that IPC-MVs transfer miR-133a-3p to H9c2 cardiomyocytes to attenuate hypoxia/reoxygenation (H/R) injury by decreasing apoptosis via targeting EGFR and suppressing the endoplasmic reticulum stress (ERS).

\section{Results}

\section{Characterization of IPC-MVs}

Transmission electron microscopy (TEM) and flow cytometry were performed to characterize circulating MVs. The morphology of both IPC-MVs and Sham-MVs observed by TEM displayed rounded vesicles surrounded by bi-layered membrane with the size ranged from 100 to $1000 \mathrm{~nm}$ (Fig. 1a-b). In flow cytometry analysis, MVs were identified as events with size less than $1 \mu \mathrm{m}$ within the gate R1 (Fig. 1c-e). Though the total amount of IPC-MVs presented a clear trend towards increasing compared with ShamMVs (Fig. 1f), there is no significant difference between these two groups. 
There were no significant differences in AAR among I/R, Sham-MV + I/R and IPC-MV + I/R groups. However, treatment with IPC-MVs, but not Sham-MVs, decreased significantly the cardiac tissue weight of IS compared with I/R group (Fig. 2a). The representative myocardial tissue staining images of four groups were shown in Fig. 2b. The IS/AAR \% was decreased significantly in IPC-MV + I/R group compared with I/R and Sham-MV + I/R groups (Fig. 2b). Meanwhile, LDH activity decreased significantly in IPC-MV group at 120 min reperfusion compared with I/R and Sham-MV + I/R groups (Fig. 2C).

\section{Protective effects of IPC-MVs on H9c2 cells against H/R injury in vitro}

To investigate the protective effects of IPC-MVs on H/R injured H9c2 cells, we treated cells with IPC-MVs or Sham-MVs. Indeed, when H9c2 cells were co-cultured with IPC-MVs, the cell viability increased significantly (Fig. 3a), and the activity of LDH decreased significantly (Fig. 3b) compared with H/R and Sham-MV + H/R groups.

\section{Differential expression of miRNAs in IPC-MVs and Sham-MVs}

MVs, acting as carrier vesicles, have intriguing role on intercellular communication through the exchange of miRNAs between cells[10]. Since MVs carry miRNAs, expression of miRNAs in circulating MVs was analyzed by microarray-based miRNA profiling. According to the results of microarray analysis, there were five differentially and over 2 fold change expressed miRNAs in IPC-MVs compared with Sham-MVs $(n=3$, Fold $\geq 2$, $P<0.05$ ) (Fig. 4a). Of total, there were 4 miRNAs (miR-1-3p, miR-378b, miR-133a-3p, miR-133b-3p) up-regulated significantly (Fold $\geq 2$ ), and 1 miRNA (miR-702-3p) down-regulated significantly (Fold $\geq 2$ ) (Fig. 4b). qRT-PCR was used to verify the result of microarray analysis. Indeed, among the miRNAs identified in circulating MVs, miR-133a-3p was the most abundant in IPC-MVs compared with Sham-MVs which was consistent with the expression pattern in microarray (Fig. 4c). Meanwhile, miR-133a-3p, a highly expressed miRNA in heart, was widely involved in the regulation of cell differentiation[19], angiogenesis[20], cardiac hypertrophy and fibrosis[21], cell apoptosis[14] and myocardial repair[22], et al. These data pointed to the miR-133a-3p as a strong candidate for the key regulatory cargo contained in IPC-MVs.

\section{Protective effects of miR-133a-3p on H9c2 cells against H/R injury}

In order to determine the effect of miR-133a-3p on cardiomyocyte apoptosis induced by H/R injury, miR133a-3p mimics or inhibitor was transfected into H9c2 cells to increase or decrease miR-133a-3p expression. As expected, after transfecting $\mathrm{H} 9 \mathrm{c} 2$ cells with miR-133a-3p mimics, there was a significant increase in cell viability, reduction in LDH activity after $\mathrm{H} / \mathrm{R}$, which was the same as treatment with IPCMVs. However, after transfecting H9c2 cells with miR-133a-3p inhibitor, there was a further decrease in cell viability, further increase in LDH activity after H/R (Fig. 5a-b). Meanwhile, detection of caspase 3 activity, and Western blot analysis of caspase 3 and cleaved-caspase 3 , was adopted to analyze the protective effect of miR-133a-3p upon apoptosis induced by H/R. Not surprisingly, miR-133a-3p mimics substantially reduced the activity of caspase 3 , and expression of caspase 3 and cleaved-caspase 3 in 
H9c2 cells, after H/R. However, miR-133a-3p inhibitor further substantially increased the activity of caspase 3 , and expression of caspase 3 and cleaved-caspase 3 in H9c2 cells, after H/R (Fig. 5c-d).

Additionally, activation of ERS markers had been detected after H/R injury in H9c2 cells, including GRP78, $\mathrm{CHOP}$ and caspase 12. As envisaged, consistent with treatment of IPC-MVs, the transfection with miR133a-3p mimics in $\mathrm{H} 9 \mathrm{c} 2$ cells significantly reduced all above proteins compared with $\mathrm{H} / \mathrm{R}$ group, whereas the expression of these proteins increased significantly after transfection with miR-133a-3p inhibitor in $\mathrm{H} 9 \mathrm{c} 2$ cells, which indicated that the suppression of ERS-induced apoptosis may be involved in the protective effect of miR-133a-3p on H/R injured H9c2 cells (Fig. 5e).

\section{HPC-MVs allow the transfer of miR-133a-3p to H9c2 cells}

Recent studies suggest that MVs transfer cardioprotective miRNAs between cardiomyocytes[13]. To conform whether MVs could transfer miR-133a-3p to H9c2 cells in vitro, we fluorescently labelled miR133a-3p mimics and transfected them into H9c2 cells. The cells were labelled with the strong red fluorescence dye Dil after $24 \mathrm{~h}$, and then the secreted MVs were collected after treatment with hypoxic preconditioning (HPC). After co-incubating H9c2 cells with fluorescently labeled HPC-MVs for $4 \mathrm{~h}$, cells exhibited red and green fluorescence in the cytoplasm (Fig. 6a), indicating that HPC-MVs derived from $\mathrm{H} 9 \mathrm{c} 2$ cells allow the transfer of miR-133a-3p to H9c2 cells. In contrast, the negative control (NC) group could not detect any fluorescence signal.

We further determined the expression level of miR-133a-3p in H9c2 cells after treated with H/R, Sham$M V s+H / R$, and IPC-MVs $+H / R$. As envisaged, qRT-PCR results showed that the expression level of miR133a-3p increased significantly when H/R injured H9c2 cells were co-cultured with IPC-MVs, but not Sham-MVs (Fig. 6b). Taken together, these results indicated that the protective effect of IPC-MVs on cardiomyocytes against $H / R$ injury may be associated with the elevation of miR-133a-3p in vitro mainly transferred by IPC-MVs.

\section{MiR-133a-3p inhibits EGFR expression in H9c2 cells}

To better understand how miR-133a-3p leads to cardioprotection, we honed in on the target genes for miR-133a-3p within IPC-MV-treated H/R injury in H9c2 cells. Among the known targets, EGFR, one of receptor tyrosine kinases (RTKs) on the cell surface, is a direct target gene in Hela cells[16] and other cancer cells[23] confirmed by Luciferase assay, qRT-PCR, and Western blot assay. According to display in KEGG database (https://www.kegg.jp/) and published literatures, we focused on the hypoxia-regulated EGFR. qRT-PCR results showed that miR-133a-3p mimics could apparent reduce, while inhibitor increase the expression of EGFR mRNA compared with NC (Fig. 7a-b). Meanwhile, Western blot results showed that miR-133a-3p mimics apparent reduce, while inhibitor increase the expression of EGFR protein compared with NC (Fig. 7c-d), which suggested that miR-133a-3p regulates the expression of EGFR in $\mathrm{H} 9 \mathrm{c} 2$ cells.

\section{MiR-133a-3p protects $H 9 c 2$ cells against $H / R$ injury through suppressing ERS}


To further investigate the underlying mechanism of the enhanced ability of miR-133a-3p in myocardial protection, AG1478, an inhibitor of EGFR, was used to pre-treat H9c2 cells before H/R. MTT results showed that AG1478 could dose-dependently increase the cell viability, and the trend became significant in $1 \mu \mathrm{M} \mathrm{AG} 1478+\mathrm{H} / \mathrm{R}$ group (Fig. 8a). We further detected LDH activity and apoptosis in H9c2 cells after treating with AG1478 $(1 \mu \mathrm{M})$. There was no doubt that the activity of LDH and caspase 3 , and protein levels of caspase 3 and cleaved-caspase 3 , reduced significantly compared with H/R group (Fig. 8b-d). Furthermore, related proteins of ERS in cells were detected including GRP78, CHOP and caspase 12. Interestingly, these proteins reduced substantially when pre-treated with AG1478 $(1 \mu \mathrm{M})$ compared with H/R group (Fig. 8e). Therefore, miR-133a-3p could be a promising therapeutic target by suppressing ERSinduced apoptosis for interventions of the protective effect of IPC-MVs upon H/R injury.

\section{Discussion}

Given the wonderful endogenous mechanism of myocardial protection, IPC has been proved to protect from myocardial I/R injury through various pathways[24]. However, the underlying molecular mechanism remains poorly understood. MVs are cystic vesicles secreted from cells under stimulation or apoptosis, which can be involved in the pathogenesis of various diseases. Recent studies have demonstrated that circulating MVs are responsible for the transmission of IPC signals that elicit cardioprotection[5, 6]. In the present study, IPC-MVs and Sham-MVs in circulation were successfully obtained, and the total amount of IPC-MVs was slightly higher than that of Sham-MVs. However, there were no significant differences in morphology, size distribution or total amount between these two groups by TEM and flow cytometry. Furthermore, our results confirmed that IPC-MVs could protect myocardium against I/R injury through reducing infarct size and LDH activity in vivo, and protect $\mathrm{H} 9 \mathrm{c} 2$ cells against $\mathrm{H} / \mathrm{R}$ injury through improving the cell viability, decreasing the LDH activity in vitro compared with Sham-MVs.

As a kind of paracrine factor, MVs allow the transfer of cytoplasmic components such as proteins, mRNAs and miRNAs from one cell to another[8]. However, circulating MVs vary in their components, which varies with the different parent cell types or different stimuli, thus playing different biological roles in occurrence and development of diseases[9, 12]. Recent studies have revealed that functional miRNAs can be transferred by MVs and appear to specially home to the site of injury within infarcted myocardium, and MVs were considered as major vector of circulating miRNAs[10]. Moreover, miRNAs have emerged as key regulators of cardiovascular physiology and diseases, including myocardial ischemic injury caused by IHD[4]. To dissect the underlying mechanisms about cardioprotective effect of IPC-MVs, microarray was performed to determine components and alterations in miRNA profiles in IPC-MVs and Sham-MVs. As expected, miRNAs could be detected in both IPC-MVs and Sham-MVs, and the types and contents of miRNAs are different. Meanwhile, we found that four miRNAs (miR-1-3p, miR-133a-3p, miR-133b-3p and miR-378b) were upregulated, and one miRNA (miR-702-3p) was downregulated, with 2-fold changes in the IPC-MVs compared with Sham-MVs. In addition, the five miRNAs mentioned above have been reported to be involved in different biological processes in cardiovascular diseases. MiR-1, a musclespecific miRNA, has been extensively studied and proven to be detrimental to cardiomyocytes[25]. MiR133 has been proved to improve cardiac function in a rat model of myocardial infarction. Mesenchymal 
stem cells with overexpressed miR-133 can reduce the infarct size and decrease the inflammatory level[22]. MiR-378 known as angio-miRNA is a key regulator of angiogenesis, which could regulate the angiogenic capacity of $\mathrm{CD} 34^{+}$progenitor cells in vivo, suggesting that this unique miRNA expression pattern represented a novel endogenous repair mechanism activated in acute myocardial infarction[26]. Moreover, overexpression of miR-378 significantly reduced intestinal epithelial cell apoptosis in ischemic models and attenuated cleaved caspase-3 expression both in vivo and in vitro[27]. MiR-702 has been identified to regulate anti-apoptotic pathway[28]. In order to verify the predictive accuracy of microarray assay, the expression of these miRNAs in IPC-MVs and Sham-MVs was detected by qRT-PCR. The results of miR-1-3p, miR-133a-3p, miR-133b-3p were consistent with the expression patterns in microarray. However, miR-378b and miR-702-3p were opposite, miR-378b was downregulated in the IPC-MVs, while miR-702-3p was upregulated compared with Sham-MVs. As a high-throughput technology, microarray is used to interpret and analyze the sequence information of samples efficiently by virtue of the hybridization and pairing characteristics of nucleic acid molecules, which is tremendously affected by background signals, and may lead to inconsistent results. Based on results of GO analysis and KEGG assay (data not shown), and multiple studies, the five miRNAs were involved in a variety of cardiovascular pathways, including aldosterone-regulated sodium reabsorption, leukocyte transendothelial migration, vasopressin-regulated water reabsorption, sphingolipid signaling pathway, phosphatidylinositol signaling system and HIF-1 signaling pathway. However, microarray and qRT-PCR assay all demonstrated that miR-133a-3p expression in the IPC-MVs increased most apparently in the detected miRNAs compared with Sham-MVs.

Increasing evidences have suggested that circulating miRNAs can be potential biomarker candidates due to their highly specific elevation in blood upon stress, including I/R injury. MiR-133a-3p plays key roles in regulating the differentiation, proliferation and maturation of cardiomyocytes. In patients with myocardial infarction (MI), the expression of miR-133a-3p in MI area decreased significantly, while increased in circulating serum/plasma[29], indicating the severity of heart injury, which demonstrated that circulating miR-133a-3p may be of clinical value as a new biomarker for the diagnosis of IHD. Moreover, multiple studies have demonstrated that miR-133a-3p can be involved in regulating a variety of cardiovascular diseases, including cardiomyopathy[30], cardiac remodeling[31], heart failure (HF)[32], and myocardial ischemic injury[33]. However, elevation of miR-133a-3p reduces hypoxia-induced, oxidative stress-induced and ERS-induced cardiomyocyte apoptosis in vitro[22]. In the present study, we further demonstrated that the decreasing of miR-133a-3p in H9c2 cells was closely related to H/R injury. When treated with IPC-MVs, miR-133a-3p expression was significantly upregulated compared with Sham-MVs. Therefore, we speculated that miR-133a-3p in IPC-MVs may mediate the protective effect of IPC-MVs on H/R-injured H9c2 cells. Consistent with the results of treatment with IPC-MVs, the ameliorative effect of miR-133a-3p on $\mathrm{H} 9 \mathrm{c} 2$ cells upon $\mathrm{H} / \mathrm{R}$ injury was also proved by the cell viability and the LDH activity detection according to the gain- and loss-of-function approaches.

Cardiomyocyte apoptosis plays central role in the progression of many myocardial disorders, including I/R injury. Here H/R-induced apoptosis was used to determine the ameliorative effect of miR-133a-3p on 
apoptosis of H9c2 cells. Consistent with the results of treatment with IPC-MVs, the ameliorative effect of miR-133a-3p on cardiomyocyte apoptosis was also proved in H/R injury detected by the expression of caspase 3 and cleaved-caspase 3 , and caspase 3 activation in $\mathrm{H} 9 \mathrm{c} 2$ cells. Our results could demonstrate the beneficial effect of miR-133a-3p on H/R-induced apoptosis.

IHD can ultimately lead to myocardial tissue death, mainly caused by cardiomyocyte apoptosis induced by tissue ischemia and hypoxia, and the trigger of myocardial injury is closely related to ERS[34, 35]. Our previous study has found that the suppression of ERS-mediated apoptosis could be involved in the protective mechanism of IPC-MVs against myocardial I/R injury, that is, the addition of IPC-MVs could counteract the I/R-induced increase of ERS-specific proteins GRP78, CHOP and caspase 12 levels[6]. Meanwhile, the importance of miR-133a-3p in cardioprotection was further supported by the finding that transfection with the miR-133a-3p mimics could suppress the ERS-induced apoptosis through reducing the expression of GRP78, CHOP and caspase 12. Instead, transfection with the miR-133a-3p inhibitor could aggravate the ERS-induced apoptosis through increasing the expression of GRP78, CHOP and caspase 12. Hence, the suppression of ERS-induced apoptosis may be involved in the protective effect of miR-133a-3p on H/R injured H9c2 cells, consistent with IPC-MVs.

Recent studies have suggested that MVs can transfer functional miRNAs between cells by various routes, including endocytotic uptaking[8]. Additionally, cardiomyocytes can release and take up MVs both in vivo and in vitro[13]. Our study further found that HPC-MVs isolated from H9c2 cells which subjected to HPC could transfer miR-133a-3p into target cells, which was verified by HPC-MVs labeled with Dil and transfected with FAM-miR-133a-3p, incubating with $\mathrm{H} 9 \mathrm{c} 2$ cells for $4 \mathrm{~h}$. Not only were $\mathrm{H} 9 \mathrm{c} 2$ cells able to take up the HPC-MVs, but the miRNA content of the HPC-MVs was also able to enter the cytoplasm.

miRNAs existing in all kinds of cells can regulate different gene expression, mainly through downregulating the expression of target genes by either promoting mRNA degradation or repressing mRNA translation. To elucidate the mechanism underlying the protective role of miR-133a-3p, we searched for the target genes by bioinformatic technology and literature search. Previous studies have shown that miR-133a-3p is mainly involved in the regulation of pro-apoptotic genes in cardiomyocytes, such as TAGLN2, DAPK2, APAF1, BCL2L11 and BMF, and so on, thus affecting the apoptosis pathways. Among known target genes, it is well known that EGFR, a hypoxia-associated apoptotic protein involving angiogenesis, is a direct target gene in cardiomyocytes[16] and many cancer cells confirmed by Luciferase assay, qRT-PCR, and Western blot. Our study further verified that EGFR is the target gene of miR-133a-3p in H9c2 cells by qRT-PCR and Western blot. Therefore, we speculated that miR-133a-3p exert a protective effect against $\mathrm{H} / \mathrm{R}$-induced apoptosis, and may be related to the inhibition of EGFR.

To further explore whether EGFR is involved in the protective mechanism of miR-133a-3p against cardiomyocyte apoptosis, H9c2 cells were pre-treated with AG1478 (an EGFR inhibitor) before H/R. We found that after treatment with AG1478 $(1 \mu \mathrm{M})$, the cell viability improved significantly, the LDH and caspase 3 activity decreased significantly, the expression of caspase 3 and cleaved-caspase 3 was downregulated significantly, and the downregulated expression of ERS-related proteins GRP78, CHOP and 
caspase 12. Therefore, the suppression of ERS-induced apoptosis might be involved in the protective mechanism of IPC-MVs on H/R injury in H9c2 cells, mediated by miR-133a-3p as evidenced by downregulating the expression of EGFR.

\section{Conclusions}

Circulating MVs after the process of IPC could convey survival signals to injured cardiomyocytes that protect cells against ERS-induced cardiomyocyte apoptosis during $H / R$ injury in vitro. The mechanism may be related to miR-133a-3p-mediated cytoprotection targeting EGFR. Furthermore, it is also suggesting that miR-133a-3p in the IPC-MVs appears as a possible novel effective strategy for the protective effect of IPC-MVs on IHD. Furthermore, the discovery of circulating MVs has shed new light on the search for more efficient miRNAs transport tools.

\section{Methods}

\section{Animals}

Healthy male Wistar rats (Beijing Vital River Laboratory Animal Technology Co., Ltd. Beijing, China) aged 8-10 weeks and weighing $253 \pm 13 \mathrm{~g}$ were used in this study. Animal experiments were approved by the Laboratory Animal Care and Use Committee of Tianjin Medical University, Tianjin, People's Republic of China and conducted according to the Guide for the Care and Use of Laboratory Animals published by the US National Institutes of Health (NIH Publication No. 85-23, revised 1996). Rats were housed in a specific animal breeding room at $24^{\circ} \mathrm{C}$ with luminosity cycles of $12 \mathrm{~h}$ light/dark and $60 \%$ relative humidity. They had been given ad libitum access to water and standard rat chow.

\section{Establishment of the I/R and IPC model in vivo}

The myocardial I/R model was established by occluding the left anterior descending (LAD) coronary artery in rats for 30 min followed by 120 min reperfusion and IPC was induced by three cycles of 5 min ischemia and 5 min reperfusion of the LAD as previously described[5]. Successful occlusions were verified by observing the development of ST-segment elevation on electrocardiogram (ECG). Shamoperated rats were received as controls.

\section{Isolation of circulating MVs}

Circulating IPC-MVs and Sham-MVs were isolated from the blood of rats subjected to IPC or Sham treatment as reported previously[6]. Briefly, healthy male Wistar rats were divided into two groups randomly with $n=5$ each: (1) Sham-MV group: rats were left untreated for $45 \mathrm{~min}$ after a silk ligature was placed around the LAD coronary artery, the blood was drawn from abdominal aorta after 45 min. (2) IPCMV group: rats were subjected to three cycles of 5 min ischemia and 5 min reperfusion of LAD after being untreated for $15 \mathrm{~min}$, then the blood was drawn from abdominal aorta at once. The blood samples in two groups were collected in sodium citrate coated tubes and centrifugated at 2,600 g, 15 min and 10,000 g, 5 
min at room temperature to obtain platelet-free plasma (PFP). Ninety $\mu \mathrm{L}$ PFP was collected and stored at $-80{ }^{\circ} \mathrm{C}$ after fixed with paraformaldehyde (PFA) to a final concentration of $1 \%$ for $1 \mathrm{~h}$ at room temperature for flow cytometry, the remaining PFP was ultracentrifuged at $100,000 \mathrm{~g}, 4^{\circ} \mathrm{C}$ for $150 \mathrm{~min}$, the supernatant was removed to obtain Sham-MVs and IPC-MVs. The pellet of MVs was resuspended in $100 \mu \mathrm{L} 0.9 \%$ sodium chloride and stored at $-80^{\circ} \mathrm{C}$. Rats were sacrificed by acute arterial hemorrhage.

\section{Transmission electron microscopy (TEM)}

TEM of circulating IPC-MVs and Sham-MVs was conducted as described previously[6]. Briefly, $40 \mu \mathrm{L} \mathrm{MV}$ resuspension was dropped on the carbon-coated copper grids, then grids were blotted dry with filter paper. For negative staining, $40 \mu \mathrm{L} 2 \%$ phosphotungstic acid ( $\mathrm{pH} 6.5)$ was used to stain for 2 min. After drying under the incandescent light, morphology of MVs was viewed in a HT7700 TEM (Hitachi, Japan), and the images were obtained using a digital camera (Olympus, Japan).

\section{Flow cytometry analysis}

Flow cytometry analysis was performed as described previously[6]. Briefly, $1 \mu \mathrm{m}$ and $2 \mu \mathrm{m}$ standard microspheres (Molecular Probe, Invitrogen, Carlsbab, CA, USA) were used to describe and calculate counts of IPC-MVs and Sham-MVs, an Accuri C6 flow cytometry (BD Biosciences, Franklin Lakes, NJ, USA) was used for analysis. Dot plots of forward scatter (FSC) versus side scatter (SSC) were established. Events $<1 \mu \mathrm{m}$ in diameter were defined as MVs. MVs could be counted by $2 \mu \mathrm{m}$ beads with known concentration. Flow cytometric results were analyzed by FlowJo 7.6 software. The absolute count of MVs was calculated with the formula: MVs $/ \mu \mathrm{L}=$ [events counted by flow cytometer' (beads added/beads counted by flow cytometer)]/sample volume.

\section{Cell culture and establishment of the H/R and hypoxic preconditioning (HPC) model of H9c2 cells in vitro}

H9c2 cells (ATCC, Manassas, VA, USA) were cultured in DMEM (Gibco, CA, USA) supplemented with $10 \%$ FBS (Gibco, CA, USA) and $1 \%$ Penicillin/Streptomycin under standard cell culture conditions $\left(37^{\circ} \mathrm{C}, 5 \%\right.$ $\mathrm{CO}_{2}$ ). All procedures were performed in accordance with the Declaration of Helsinki of the World Medical Association and the research protocol was approved by Ethics Committee of Tianjin Medical University.

For the $\mathrm{H} / \mathrm{R}$ injury model, $\mathrm{H} 9 \mathrm{c} 2$ cells were stimulated by $\mathrm{H} / \mathrm{R}$ as previously described[17]. Briefly, $\mathrm{H} 9 \mathrm{c} 2$ cells were subjected to hypoxic buffer (in mM: $0.9 \mathrm{NaH}_{2} \mathrm{PO}_{4}, 6.0 \mathrm{NaHCO}_{3}, 1.0 \mathrm{CaCl}_{2}, 1.2 \mathrm{MgSO}_{4}, 98.5$ $\mathrm{NaCl}, 10.0 \mathrm{KCl}, 20.0 \mathrm{HEPES}, 40.0$ sodium lactate, $\mathrm{pH} 6.2)$ in a hypoxic chamber $\left(95 \% \mathrm{~N}_{2}-5 \% \mathrm{CO}_{2}\right.$, BillupsRothenberg, Del Mar, CA, USA) in $37^{\circ} \mathrm{C}$ for $12 \mathrm{~h}$ followed by reoxygenation under standard cell culture conditions for $4 \mathrm{~h}$. The control group was maintained in control buffer (in mM: $0.9 \mathrm{NaH}_{2} \mathrm{PO}_{4}, 20.0$ $\mathrm{NaHCO}_{3}, 1.0 \mathrm{CaCl}_{2}, 1.2 \mathrm{MgSO}_{4}, 129.5 \mathrm{NaCl}, 5.0 \mathrm{KCl}, 20.0$ HEPES, 5.5 glucose, $\mathrm{pH} 7.4$ ) under standard cell culture conditions for $16 \mathrm{~h}$.

For the HPC model, H9c2 cells were transferred between hypoxic and normoxic conditions for five cycles as described previously[18]. Briefly, cells were placed into the hypoxic chamber under hypoxic buffer (pH 
6.8) incubating with $95 \% \mathrm{~N}_{2}-5 \% \mathrm{CO}_{2}$ for 10 min, then were cultured normally in $37^{\circ} \mathrm{C}, 5 \% \mathrm{CO}_{2}$ for 15 $\min$.

\section{Cell transfection}

The FAM-miR-133a-3p mimics or miR-133a-3p mimics, inhibitor and negative control (NC) miRNAs (GenePharma, Shanghai, China) were transfected into H9c2 cells using Lipofectamine 2000 (Invitrogen, USA) according to the manufacturer's instructions. The work concentration of mimics and mimics NC was $50 \mathrm{nM}$, whereas that of inhibitor and inhibitor NC was $100 \mathrm{nM}$. The transfection medium was refreshed after $6 \mathrm{~h}$, and cells were harvested for total RNAs and proteins extraction after being cultured for $24 \mathrm{~h}$. The efficiency of mimics or inhibitor was confirmed by qRT-PCR. Following that, the level of EGFR mRNA and the expression of EGFR protein were detected by qRT-PCR and Western blot, respectively. Meanwhile, after being transfected for $24 \mathrm{~h}, \mathrm{H} 9 \mathrm{c} 2$ cells were treated with H/R injury for further study.

\section{Treatment with IPC-MVs on I/R injured rats in vivo}

Rats were anesthetized intraperitoneally by $25 \%$ ethyl carbamate, then performed a thoracotomy before ligation of LAD coronary artery. The ends of the silk suture were passed through a polyethylene tube, which was used to occlude the LAD by pulling the thread. After achieving hemodynamic stability for 15 min, all rats were divided into four groups randomly with $n=5$ each. (1) Sham group, rats were left untreated for 145 min after a silk ligature was placed around the LAD. (2) I/R group, rats received I/R process. (3) Sham-MV + I/R group, Sham-MVs (7 mg/ $\mathrm{kg}$ ) were infused via the femoral vein in I/R injured rats. (4) IPC-MV + I/R group, IPC-MVs (7 mg/ $\mathrm{kg}$ ) were infused via the femoral vein in I/R injured rats. The same volume of $0.9 \%$ sodium chloride was given to Sham and I/R groups. All treatments began at $25-\mathrm{min}$ ischemia, with additional 1 min infusion.

\section{Treatment with IPC-MVs on H/R injured H9c2 cells in vitro}

H9c2 cells were cultured under normal condition for $24 \mathrm{~h}$, and were divided into four groups. (1) Control group, control buffer ( $\mathrm{pH}$ 7.4) was added. (2) H/R group, hypoxic buffer ( $\mathrm{pH}$ 6.2) was added. (3) Sham$\mathrm{MV}+\mathrm{H} / \mathrm{R}$ group, hypoxic buffer $(\mathrm{pH}$ 6.2) with Sham-MVs $(100 \mu \mathrm{g} / \mathrm{mL})$ was added. (4) IPC-MV + H/R group, hypoxic buffer $(\mathrm{pH}$ 6.2) with IPC-MVs $(100 \mu \mathrm{g} / \mathrm{mL})$ was added. H9c2 cells in all groups were exposed to $12 \mathrm{~h}$ of hypoxia and $4 \mathrm{~h}$ of reoxygenation except Control group.

\section{Measurement of myocardial infarct size}

Hearts were ligated at 120 min after reperfusion, before the injection of $0.6 \%$ trypan blue solution via femoral vein, then cut off immediately. Hearts were washed in normal saline (NS) before frozen at $-20{ }^{\circ} \mathrm{C}$ for $30 \mathrm{~min}$, sliced into 1-mm-thick sections transversely from apex to base, and then incubated with $1 \%$ TTC (Sigma-Aldrich, St. Louis, MO, USA) at $37^{\circ} \mathrm{C}$ for $15 \mathrm{~min}$ and finally incubated with $10 \%$ formalin solution for $24 \mathrm{~h}$. The infarct size (IS) was gray and the area at risk (AAR) was brick red. IS and AAR were 
isolated and weighted, respectively, then the ratio of IS to AAR (IS/AAR\%) was calculated as the infarct size.

\section{Cell viability assay}

Cell viability was determined using methyl thiazolyl tetrazolium (MTT, Solarbio, Beijing, China) method. After treatment with H/R injury, H9c2 cells were incubated with $10 \mu \mathrm{L} 0.5 \%$ MTT solution for $4 \mathrm{~h}$ at $37^{\circ} \mathrm{C}$. Then the supernatant was discarded and $150 \mu \mathrm{L}$ dimethyl sulfoxide was added to each well. Absorbance was measured at a wavelength of $490 \mathrm{~nm}$ with a microplate reader (Bio-Rad Laboratories, CA, USA) after the culture plate was shaken at high speed for $10 \mathrm{~min}$.

\section{LDH activity assay}

The $0.2 \mathrm{~mL}$ blood was drawn from femoral vein at reperfusion $120 \mathrm{~min}$, respectively, then centrifuged at $3000 \mathrm{~g}$ at room temperature for $15 \mathrm{~min}$ to obtain supernatant. After treatment with H/R injury, H9c2 cell culture supernatant was collected. All the supernatants were measured using LDH assay kit (Jiancheng Bioengineering Institute, Nanjing, China) according to the manufacturer's instruction.

\section{Caspase 3 activity assay}

For detection of the activity of caspase $3, \mathrm{H} 9 \mathrm{c} 2$ cells were lysed at $4{ }^{\circ} \mathrm{C}$ for $15 \mathrm{~min}$ by caspase 3 lysis buffer (Beyotime, Nanjing, China). Protein extracts of $50 \mu \mathrm{L}$ were incubated with $50 \mu \mathrm{L}$ reaction buffer containing caspase 3 substrate (Ac-DEVD-pNA, $2 \mathrm{mM}$ ) for $2 \mathrm{~h}$ at $37^{\circ} \mathrm{C}$. The absorbance was measured at a wavelength of $405 \mathrm{~nm}$ with a multilabel reader (Bio-Tek, Winooski, VT, USA). Caspase 3 activity in H9c2 cells was calculated using the standard curve.

\section{Microarray analysis of circulating MVs}

Total RNAs were obtained from the six MV samples (IPC-MVs, $n=3$, Sham-MVs, $n=3$ ), then were quantified by the NanoDrop ND-2000 (Thermo Scientific) and the RNA integrity was detected by Agilent Bioanalyzer 2100 (Agilent Technologies). After all samples passed the quality control analysis, the total RNAs were dephosphorylated, denaturated and then labeled with Cyanine-3-CTP. After purification, the labeled RNAs were hybridized onto the microarray. After being washed, the arrays were scanned using the Agilent Scanner G2505C (Agilent Technologies).

\section{Isolation and quantification of miRNAs from circulating MVs}

Total RNAs were extracted from MVs using the miRNeasy kit (Qiagen) according to the manufacturer's instruction. The yield of RNAs was determined using NanoDrop 2000 (Thermo Scientific, USA), and integrity evaluated using agarose gel electrophoresis. Then reverse transcription was performed with miScript Reverse Transcriptase Kit (Qiagen, Germany) and quantification of miRNAs was performed with QuantiFast ${ }^{\circledR}$ SYBR ${ }^{\circledR}$ Green PCR Kit (Qiagen, Germany). Amplification was performed at $95^{\circ} \mathrm{C}$ for 10 min, followed by $30 \sim 45$ cycles of $95^{\circ} \mathrm{C}$ for $10 \mathrm{~s}, 60^{\circ} \mathrm{C}$ for $20 \mathrm{~s}$ and $72^{\circ} \mathrm{C}$ for $15 \mathrm{~s}$. Each sample was run in 
triplicate for analysis. miRNA primers were subscribed from Generay Biotech (Generay, PRC), and the levels of miRNA analyzed by qRT-PCR were normalized to that of U6 snRNA. Fold induction was calculated using the $\mathrm{Ct}$ method: $\mathrm{DDCt}=\left(\mathrm{Ct}_{\text {Target miRNA }}-\mathrm{Ct}_{\mathrm{U} 6}\right)$ IPC-MVs - $\left(\mathrm{Ct}_{\text {Target miRNA }}-\mathrm{Ct}_{\mathrm{U} 6}\right) \mathrm{Sham}-\mathrm{MVs}$, and the final data were derived from $2^{-D D C t}$.

\section{HPC-MV preparation and uptake by $\mathrm{H} 9 \mathrm{c} 2$ cells}

For generating HPC-MVs from miR-133a-3p overexpressed H9c2 cells, the FAM-miR-133a-3p mimics were transfected into cells using Lipofectamine 2000. After $24 \mathrm{~h}, \mathrm{H} 9 \mathrm{c} 2$ cells were labeled with a fluorescent dye Dil (Beyotime, Nanjing, China) by incubating them in the Dil working solution $(5 \mu \mathrm{M})$ for $10 \mathrm{~min}$ at $37^{\circ} \mathrm{C}$, followed by washing with D-hank's solution. And then HPC-MVs were collected from the hypoxic buffer of H9c2 cells after treatment with HPC as described above. Briefly, hypoxic buffer was centrifuged at $2700 \mathrm{~g}, 4^{\circ} \mathrm{C}$ for $20 \mathrm{~min}$ to remove cell debris, followed by ultracentrifugation at $100,000 \mathrm{~g}, 4^{\circ} \mathrm{C}$ for 148 min to obtain HPC-MVs. The pellet was resuspended in $50 \mu \mathrm{L}$ D-hank's solution and stored at $-80^{\circ} \mathrm{C}$. The protein concentration of MVs was determined by BCA assay (Beyotime, Nanjing, China). Then HPC-MVs $\left(30 \mu \mathrm{g} / \mathrm{mL}\right.$ ) were incubated with $\mathrm{H} 9 \mathrm{c} 2$ cells for $4 \mathrm{~h}$ at $37^{\circ} \mathrm{C}, 4 \%$ paraformaldehyde (Solarbio, Beijing) was used for fixing cells, DAPI was used for nucleus staining, and images were collected using a fluorescence microscope (Leica, Germany). HPC-MVs derived from unlabeled H9c2 cells were used as negative control (NC).

\section{qRT-PCR}

Cellular RNAs of each group were extracted using Trizol reagent (Invitrogen, USA). The purity of RNAs was determined by OD260/280 using a Nanodrop 2000 (DeNovix, USA), and integrity evaluated using agarose gel electrophoresis. The specific stem-looped qRT-PCR primers for miR-133a-3p were designed by GenePharma Co.Ltd. (Shanghai, China) (The sequences of rno-miR-133a-3p primers were: forward, ATGCTCATTTGGTCCCCTTC; reverse, TATGGTTGTTCTGCTCTCTGTCTC). EGFR and GAPDH primers were subscribed from GENEWIZ (Suzhou, China) (The sequences of rat EGFR primers were: forward, 5'TCCAACTTTTACCGAGCCCT-3'; reverse, 5'-TCAAGAGTGGAGTCCGTGAC-3'. The sequences of rat GAPDH primers were: forward, 5'-TCGTGGAGTCTACTGGCGTCTT-3'; reverse, 5'- CATTGCTGACAATCTTGAGGGAG$3^{\prime}$ ). Mature miR-133a-3p levels were measured using SYBR Green Realtime PCR Master Mix (TIANGEN, Beijing, China) according to the manufacturer's instructions. The stem-loop cDNAs were generated from 1 $\mu \mathrm{g}$ of RNAs using FastQuant cDNA First Chain Synthesis Kit (TIANGEN, Beijing, China). Reverse transcription was performed at $42^{\circ} \mathrm{C}$ for $3 \mathrm{~min}$, followed by $42^{\circ} \mathrm{C}$ for $15 \mathrm{~min}, 95^{\circ} \mathrm{C}$ for $3 \mathrm{~min}$. Then qRTPCR was carried out on a CFX96 Real-time System instrument (Bio-Rad, USA). Amplification was performed at $95^{\circ} \mathrm{C}$ for $15 \mathrm{~min}$, followed by 40 cycles of $95^{\circ} \mathrm{C}$ for $10 \mathrm{~s}, 60^{\circ} \mathrm{C}$ for $32 \mathrm{~s}$. miR-133a-3p and U6 primers were subscribed from GenePharma (Shanghai, China), and the levels of miR-133a-3p analyzed by qRT-PCR were normalized to that of U6 snRNA.

Quantitative detection of EGFR mRNA was also performed by qRT-PCR as described above, and the mRNAs of GAPDH were used for normalization. Each sample was run in triplicate for analysis. Relative 
expression of miR-133a-3p or EGFR was calculated using the $2^{-D D C t}$ method.

\section{Western blot}

Total proteins were extracted from H9c2 cells of each group with the Western and IP lysis buffer (Beyotime, Nanjing, China) for Western blot. Protein lysates were then centrifuged at 12,000 g for 10 min at $4^{\circ} \mathrm{C}$. Total protein quantification was performed using a BCA protein assay. Equal amounts of proteins $(20 \mu \mathrm{g})$ were separated by $8 \%$ or $10 \%$ SDS electrophoresis, and transferred onto PVDF membranes. Blots were blocked by $5 \%$ skim milk for $2 \mathrm{~h}$ at room temperature, then incubated with primary anti-EGFR (Immunoway, USA, 1: 1000), anti-caspase 3 (Cell Signaling Technology, Danvers, MA, USA; 1:1000 dilutions), anti-cleaved caspase 3 (Cell Signaling Technology, Danvers, MA, USA; 1:1000 dilutions), antiGRP78 (Cell Signaling Technology, Danvers, MA, USA; 1:1000 dilutions), anti-CHOP (Santa Cruz Biotechnology, Inc., CA, USA; 1:500 dilution), anti-caspase 12 (Santa Cruz Biotechnology, Inc., CA, USA; 1:500 dilutions) or anti-b-actin (Cell Signaling Technology, Danvers, MA, USA; 1:1000 dilutions) antibodies overnight at $4{ }^{\circ} \mathrm{C}$, followed by horseradish peroxidase (HRP)-conjugated secondary antibody (Beyotime, Nanjing, China; 1:1000 dilutions) for $2 \mathrm{~h}$, then after chemiluminescent ECL detection (Beyotime, Nanjing, China), analyzed with ImageJ software (NIH, Bethesda MD).

\section{Statistical analysis}

The raw data of microarray were analyzed by Feature Extraction software (version 10.7.1.1, Agilent Technologies). Genespring software (version 14.8, Agilent Technologies) was employed to finish the basic analysis with the raw data. Differentially expressed miRNAs were then identified through fold change and $\mathrm{P}$ value calculated using t-test. The threshold set for up- and down-regulated genes was a fold change $\geq 2.0$ and a $P$ value $\leq 0.05$.

All values were expressed as Mean \pm standard derivation (SD). An unpaired Student's t-test was used for statistical analysis between two groups, and one-way analysis of variance (ANOVA) followed by the Tukey post hoc tests was used for multiple groups $(>2)$. $P<0.05$ was considered significant. Statistical analyses were performed using SPSS 17.0.

\section{Abbreviations}

\section{AAR}

area at risk; ANOVA:one-way analysis of variance; ECG:electrocardiogram; EGFR:epidermal growth factor receptor; ERS:endoplasmic reticulum stress; EVs:extracellular vesicles; FSC:forward scatter; HF:heart failure; hMSCs:human mesenchymal stem cells; HPC:hypoxic preconditioning;

H/R:hypoxia/reoxygenation; IHD:ischemic heart disease; IPC:ischemic preconditioning; IPC-MVs:ischemic preconditioning-derived microvesicles; I/R:ischemia/reperfusion; IS:infarct size; LAD:left anterior descending artery; LDH:lactate dehydrogenase; MI:myocardial infarction; miRNAs:microRNAs; 
MTT:methyl thiazolyl tetrazolium; MVs:microvesicles; NC:negative control; PFA:paraformaldehyde; PFP:platelet-free plasma; RTKs:receptor tyrosine kinases; SD:standard derivation; SSC:side scatter; TEM:transmission electron microscopy.

\section{Declarations}

\section{Ethics approval and consent to participate}

All procedures were performed in accordance with the Declaration of Helsinki of the World Medical Association and the research protocol was approved by Ethics Committee of Tianjin Medical University.

\section{Consent to publish}

All authors have read and approved the submission of this manuscript.

\section{Availability of data and materials}

The data sets used and/or analyzed during the current study are available from the corresponding author on reasonable request.

\section{Competing Interests}

The authors declare that they have no conflict of interest.

\section{Funding}

This work was supported by the Specialized Research Fund for Doctoral Program of Higher Education of China (grant mumber 20101202110005), the Natural Science Fund of Tianjin Education Commission for Higher Education (grant numbers 20110106), and the Natural Science Foundation of Tianjin City (grant numbers 11JCZDJC18300).

\section{Authors' Contributions}

All authors conceived and designed the study; JYZ and QZ conducted the experiments, collected and analyzed the data. JYZ wrote the first draft of the manuscript. All authors contributed to and approved the final manuscript. 


\section{Acknowledgements}

Not Applicable.

\section{References}

1.

Ghaderi S, Alidadiani N, Dilaver N, Heidari HR, Parvizi R, Rahbarghazi R, Soleimani-Rad J, Baradaran B. Role of glycogen synthase kinase following myocardial infarction and ischemia-reperfusion. Apoptosis. 2017;22(7):887-97.

2.

Wen MH, Gong ZJ, Huang CH, Liang Q, Xu MX, Wang LQ, Zhang WF, Lu P, Zhan BM, Yu LL, et al. Plasma exosomes induced by remote ischaemic preconditioning attenuate myocardial ischaemia/reperfusion injury by transferring miR-24. Cell Death Dis. 2018;9(3):320.

3.

Borosch S, Dahmen E, Beckers C, Stoppe C, Buhl EM, Denecke B, Goetzenich A, Kraemer S. Characterization of extracellular vesicles derived from cardiac cells in an in vitro model of preconditioning. J Extracell Vesicles. 2017;6(1):1390391.

4.

Song MA, Paradis AN, Gay MS, Shin J, Zhang L. Differential expression of microRNAs in ischemic heart disease. Drug Discov Today. 2015;20(2):223-35.

5 .

Wang Y, Wei S, Wang YL, Liu M, Shang M, Zhang Q, Wu YN, Liu ML, Song JQ, Liu YX. Protective effects of circulating microvesicles derived from myocardial ischemic rats on apoptosis of cardiomyocytes in myocardial ischemia/reperfusion injury. Oncotarget. 2017;8(33):54572-82.

6.

Liu M, Wang Y, Zhu Q, Zhao J, Wang Y, Shang M, Liu M, Wu Y, Song J, Liu Y. Protective effects of circulating microvesicles derived from ischemic preconditioning on myocardial ischemia/reperfusion injury in rats by inhibiting endoplasmic reticulum stress. Apoptosis. 2018;23(7-8):436-48.

7.

Chen Y, Li G, Liu ML. Microvesicles as emerging biomarkers and therapeutic targets in cardiometabolic diseases. Genomics Proteomics Bioinformatics. 2018;16(1):50-62.

8.

Liu Y, Li Q, Hosen MR, Zietzer A, Flender A, Levermann P, Schmitz T, Fruhwald D, Goody P, Nickenig G, et al. Atherosclerotic conditions promote the packaging of functional microRNA-92a-3p into endothelial microvesicles. Circ Res. 2019;124(4):575-87.

9.

Luo H, Li X, Li T, Zhao L, He J, Zha L, Qi Q, Yu Z. microRNA-423-3p exosomes derived from cardiac fibroblasts mediates the cardioprotective effects of ischaemic post-conditioning. Cardiovasc Res. 
2019;115(7):1189-204.

10.

Cheng M, Yang J, Zhao X, Zhang E, Zeng Q, Yu Y, Yang L, Wu B, Yi G, Mao X, et al. Circulating myocardial microRNAs from infarcted hearts are carried in exosomes and mobilise bone marrow progenitor cells. Nat Commun. 2019;10(1):959.

11.

Mellis D, Caporali A. MicroRNA-based therapeutics in cardiovascular disease: screening and delivery to the target. Biochem Soc Trans. 2018;46(1):11-21.

12.

Li H, Liao Y, Gao L, Zhuang T, Huang Z, Zhu H, Ge J. Coronary serum exosomes derived from patients with myocardial ischemia regulate angiogenesis through the miR-939-mediated nitric oxide signaling pathway. Theranostics. 2018;8(8):2079-93.

13.

Yang Y, Li Y, Chen X, Cheng X, Liao Y, Yu X. Exosomal transfer of miR-30a between cardiomyocytes regulates autophagy after hypoxia. J Mol Med (Berl). 2016;94(6):711-24.

14.

Li S, Xiao FY, Shan PR, Su L, Chen DL, Ding JY, Wang ZQ. Overexpression of microRNA-133a inhibits ischemia-reperfusion-induced cardiomyocyte apoptosis by targeting DAPK2. J Hum Genet. 2015;60(11):709-16.

15.

Li AY, Yang Q, Yang K. miR-133a mediates the hypoxia-induced apoptosis by inhibiting TAGLN2 expression in cardiac myocytes. Mol Cell Biochem. 2015;400(1-2):173-81.

16.

Lee SY, Ham O, Cha MJ, Song BW, Choi E, Kim IK, Chang W, Lim S, Lee CY, Park JH, et al. The promotion of cardiogenic differentiation of hMSCs by targeting epidermal growth factor receptor using microRNA133a. Biomaterials. 2013;34(1):92-9.

17.

Zhang Q, Shang M, Zhang M, Wang Y, Chen Y, Wu Y, Liu M, Song J, Liu Y. Microvesicles derived from hypoxia/reoxygenation-treated human umbilical vein endothelial cells promote apoptosis and oxidative stress in H9c2 cardiomyocytes. BMC Cell Biol. 2016;17(1):25.

18.

Yu H, Yang Z, Pan S, Yang Y, Tian J, Wang L, Sun W. Hypoxic preconditioning promotes the translocation of protein kinase $C$ epsilon binding with caveolin-3 at cell membrane not mitochondrial in rat heart. Cell Cycle. 2015;14(22):3557-65.

19.

Wust S, Drose S, Heidler J, Wittig I, Klockner I, Franko A, Bonke E, Gunther S, Gartner U, Boettger T, et al. Metabolic maturation during muscle stem cell differentiation is achieved by miR-1/133a-mediated inhibition of the Dlk1-Dio3 mega gene cluster. Cell Metab. 2018;27(5):1026-39.

20. 
Izarra A, Moscoso I, Levent E, Canon S, Cerrada I, Diez-Juan A, Blanca V, Nunez-Gil IJ, Valiente I, Ruiz-Sauri A, et al. MiR-133a enhances the protective capacity of cardiac progenitors cells after myocardial infarction. Stem Cell Rep. 2014;3(6):1029-42.

21.

Diniz GP, Lino CA, Guedes EC, Moreira LN, Barreto-Chaves ML. Cardiac microRNA-133 is down-regulated in thyroid hormone-mediated cardiac hypertrophy partially via Type 1 Angiotensin II receptor. Basic Res Cardiol. 2015;110(5):49.

22.

Chen Y, Zhao Y, Chen W, Xie L, Zhao ZA, Yang J, Chen Y, Lei W, Shen Z. MicroRNA-133 overexpression promotes the therapeutic efficacy of mesenchymal stem cells on acute myocardial infarction. Stem Cell Res Ther. 2017;8(1):268.

23.

Tang Y, Pan J, Huang S, Peng X, Zou X, Luo Y, Ren D, Zhang X, Li R, He P, et al. Downregulation of miR133a-3p promotes prostate cancer bone metastasis via activating PI3K/AKT signaling. J Exp Clin Cancer Res. 2018;37(1):160.

24.

Castaldo P, Macri ML, Lariccia V, Matteucci A, Maiolino M, Gratteri S, Amoroso S, Magi S. $\mathrm{Na}^{+} / \mathrm{Ca}^{2+}$ exchanger 1 inhibition abolishes ischemic tolerance induced by ischemic preconditioning in different cardiac models. Eur J Pharmacol. 2017;794:246-56.

25.

Kang B, Li W, Xi W, Yi Y, Ciren Y, Shen H, Zhang Y, Jiang H, Xiao J, Wang Z. Hydrogen sulfide protects cardiomyocytes against apoptosis in ischemia/reperfusion through miR-1-regulated histone deacetylase 4 pathway. Cell Physiol Biochem. 2017;41(1):10-21.

26.

Templin C, Volkmann J, Emmert MY, Mocharla P, Muller M, Kraenkel N, Ghadri JR, Meyer M, StypRekowska B, Briand S, et al. Increased proangiogenic activity of mobilized CD34 + progenitor cells of patients with acute ST-segment-elevation myocardial infarction: role of differential microRNA-378 expression. Arterioscler Thromb Vasc Biol. 2017;37(2):341-9.

27.

Li Y, Wen S, Yao X, Liu W, Shen J, Deng W, Tang J, Li C, Liu K. MicroRNA-378 protects against intestinal ischemia/reperfusion injury via a mechanism involving the inhibition of intestinal mucosal cell apoptosis. Cell Death Dis. 2017;8(10):e3127.

28.

Zhang WG, Chen L, Dong Q, He J, Zhao HD, Li FL, Li H. Mmu-miR-702 functions as an anti-apoptotic mirtron by mediating ATF6 inhibition in mice. Gene. 2013;531(2):235-42.

29.

Kuwabara Y, Ono K, Horie T, Nishi H, Nagao K, Kinoshita M, Watanabe S, Baba O, Kojima Y, Shizuta S, et al. Increased microRNA-1 and microRNA-133a levels in serum of patients with cardiovascular disease indicate myocardial damage. Circ Cardiovasc Genet. 2011;4(4):446-54.

30 . 
Rubis P, Toton-Zuranska J, Wisniowska-Smialek S, Holcman K, Kolton-Wroz M, Wolkow P, Wypasek E, Natorska J, Rudnicka-Sosin L, Pawlak A, et al. Relations between circulating microRNAs (miR-21, miR-26, miR-29, miR-30 and miR-133a), extracellular matrix fibrosis and serum markers of fibrosis in dilated cardiomyopathy. Int J Cardiol. 2017;231:201-6.

31.

Li N, Zhou H, Tang Q. MiR-133: a suppressor of cardiac remodeling? Front Pharmacol. 2018;9:903. 32.

Sang HQ, Jiang ZM, Zhao QP, Xin F. MicroRNA-133a improves the cardiac function and fibrosis through inhibiting Akt in heart failure rats. Biomed Pharmacother. 2015;71:185-9.

33.

Yu SY, Dong B, Tang L, Zhou SH. LncRNA MALAT1 sponges miR-133 to promote NLRP3 inflammasome expression in ischemia-reperfusion injured heart. Int J Cardiol. 2018;254:50.

34.

Ren L, Wang Q, Chen Y, Ma Y, Wang D. Involvement of microRNA-133a in the protective effect of hydrogen sulfide against ischemia/reperfusion-induced endoplasmic reticulum stress and cardiomyocyte apoptosis. Pharmacology. 2019;103(1-2):1-9.

35.

Xie F, Wu D, Huang SF, Cao JG, Li HN, He L, Liu MQ, Li LF, Chen LX. The endoplasmic reticulum stressautophagy pathway is involved in apelin-13-induced cardiomyocyte hypertrophy in vitro. Acta Pharmacol Sin. 2017;38(12):1589-600.

\section{Figures}


a

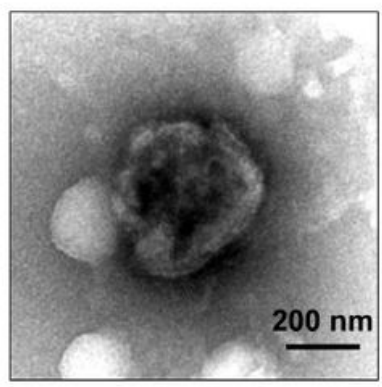

d

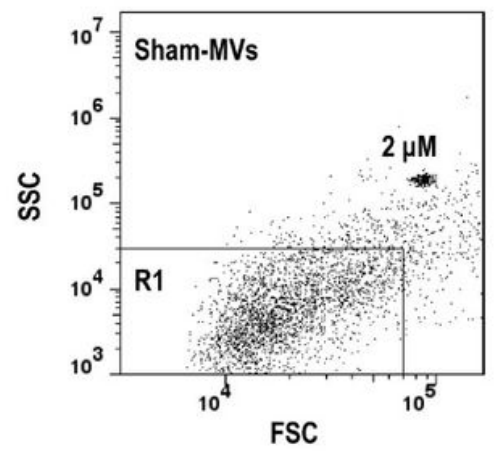

b

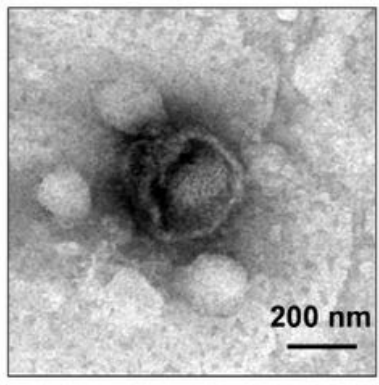

e

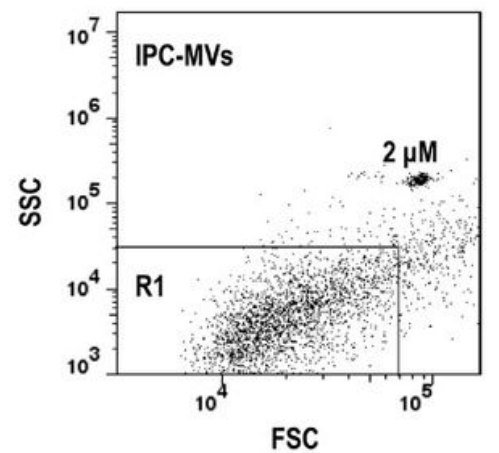

C

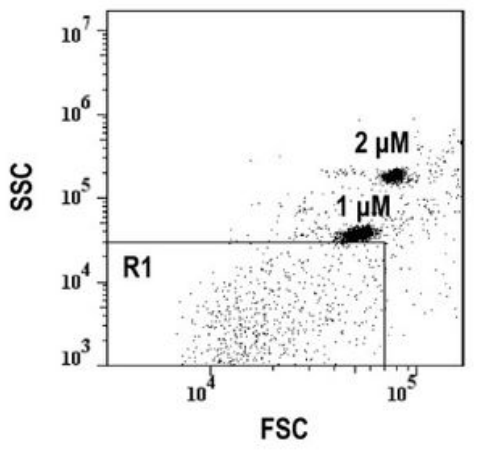

f

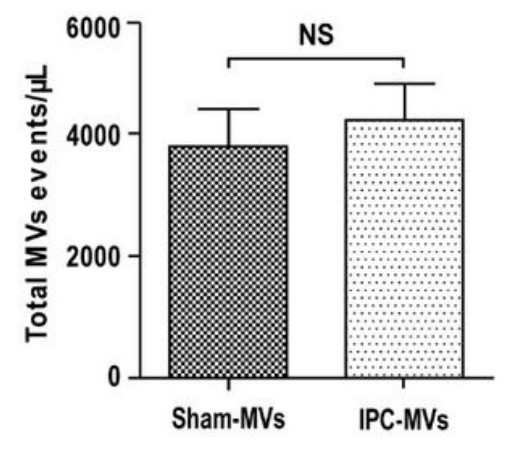

\section{Figure 1}

Characterization of circulating IPC-MVs by transmission electron microscopy (TEM) and flow cytometry. a-b Photo of Sham-MVs (a) and IPC-MVs (b) by TEM. c-e Representative dot plots of forward scatter (FSC) vs. side scatter (SSC) for evaluation of 1,2 $\mu \mathrm{m}$ calibration beads (c), Sham-MVs (d) and IPC-MVs (e) were identified as events with size less than $1 \mu \mathrm{m}$ within the gate R1. f Effect of IPC treatment on total amount of circulating MVs in rats; NS means no significant differences, $n=5$ 
a

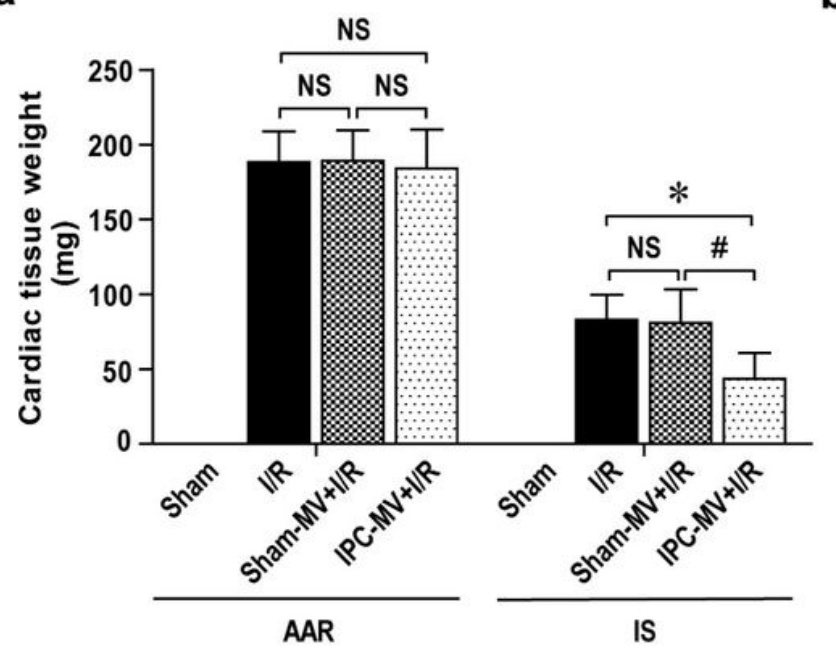

b

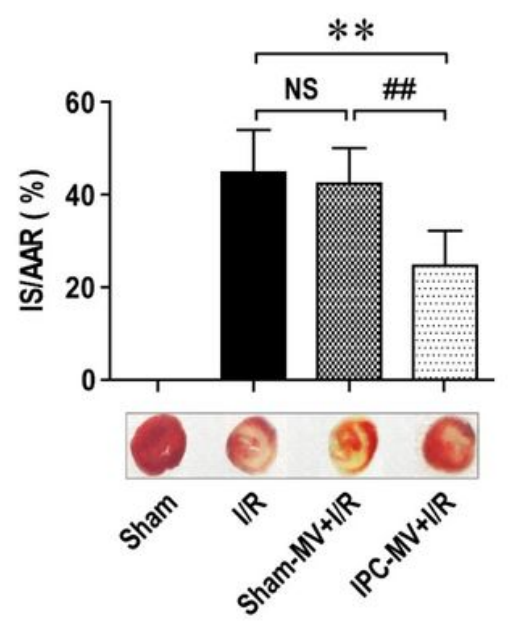

C

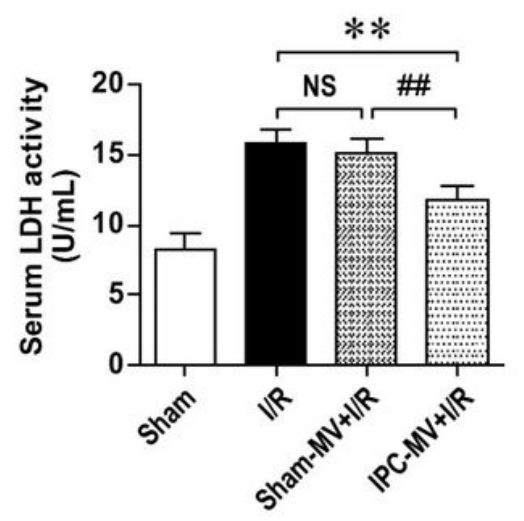

Figure 2

Protective effects of IPC-MVs on myocardial I/R injury in vivo. a-b Effects of IPC-MVs on myocardial infarct size in I/R injured rats. Cardiac tissue weight of IS and AAR (a), ratio of IS to AAR (IS/AAR \%), and the representative photographs of myocardial tissue sections (The gray areas represent infarct) (b). $c$ Serum LDH activity assay. ${ }^{*} \mathrm{P}<0.05$, ${ }^{\star} \mathrm{P}<0.01$ vs. I/R; \#P<0.05, \#\#P<0.01 vs. Sham-MV + I/R; NS means no significant differences, $n=5$

a

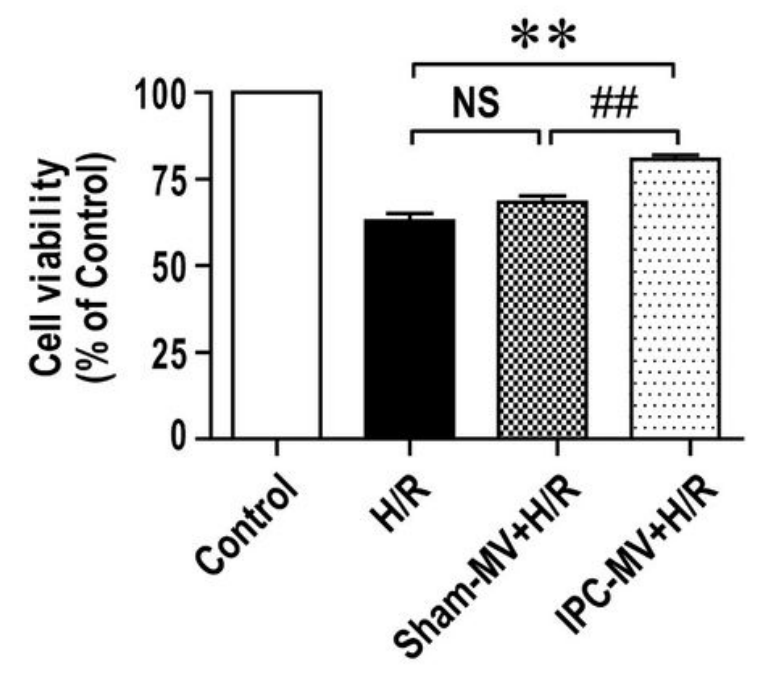

b

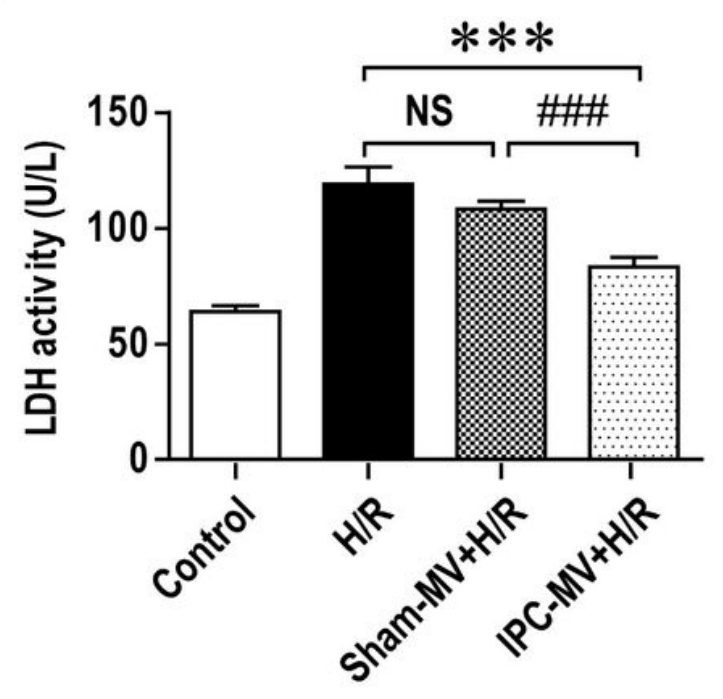

\section{Figure 3}

Protective effects of IPC-MVs on H9c2 cells against H/R injury. a Cell viability assay (MTT). b LDH activity assay. ${ }^{* * P}<0.01,{ }^{* \star *} P<0.001$ vs. I/R; \#\#P<0.01, \#\#\#P<0.001 vs. Sham-MV + I/R; NS means no 
significant differences, $\mathrm{n}=6$

a

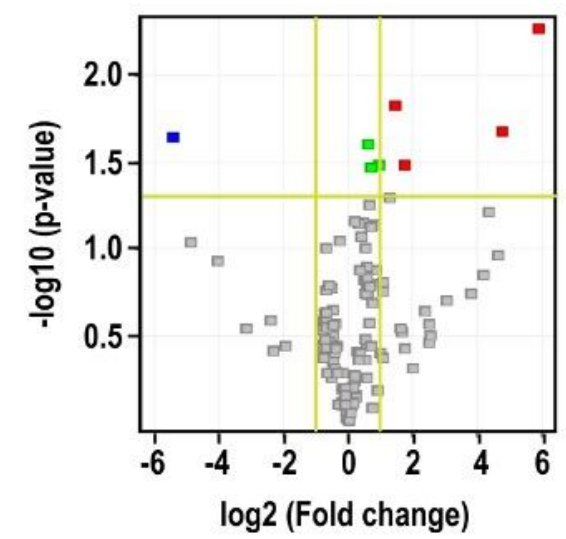

b

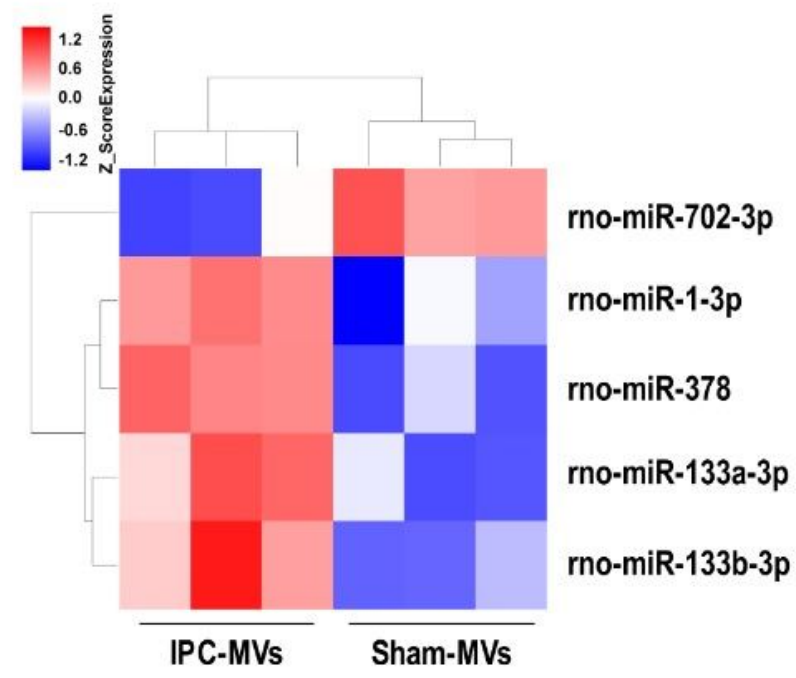

C

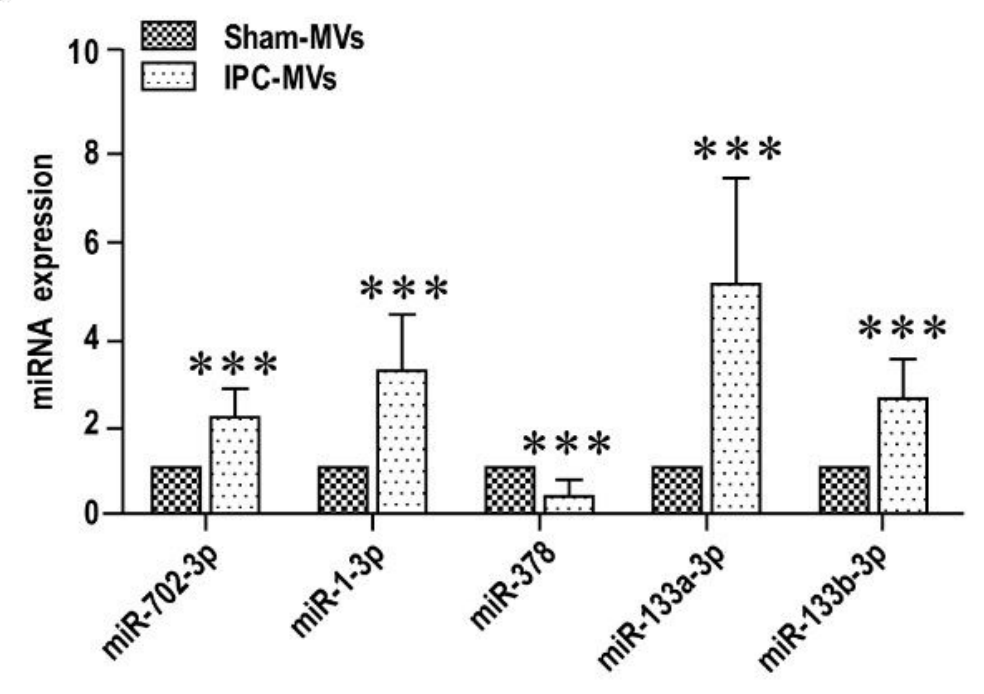

Figure 4

MiRNA expression profile of IPC-MVs and Sham-MVs. a Volcano plot revealing the effect of IPC treatment on miRNA expression in circulating MVs. b Cluster analysis of 5 miRNAs with significantly differential expression in IPC-MVs. miRNAs up-regulated in IPC-MVs were labeled in red and the one down-regulated was labeled in blue compared to Sham-MVs. c Expression of 5 miRNAs with significantly differential expression in IPC-MVs detected by qRT-PCR (normalized to U6 snRNA). ${ }^{* \star * P}<0.001$ vs. Sham-MVs; $n=3$ 
a

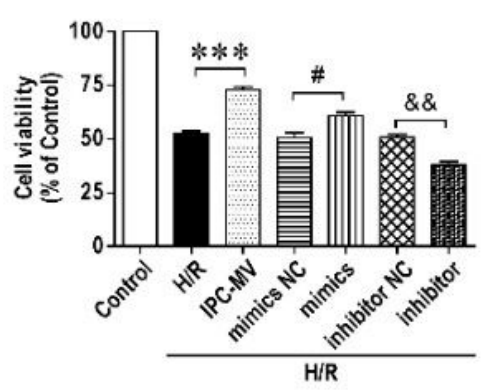

d

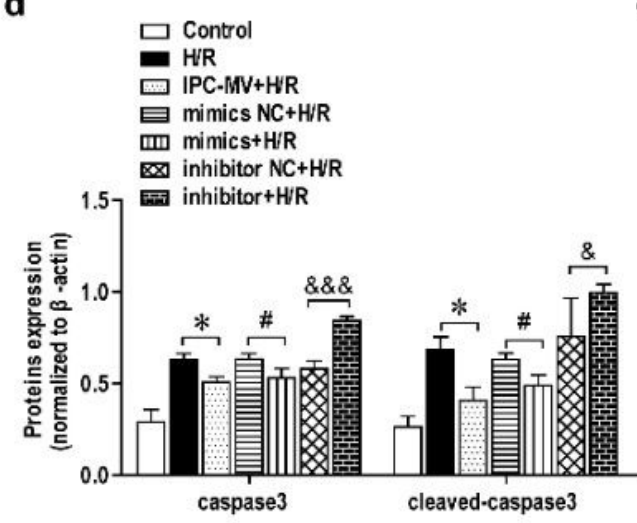

b

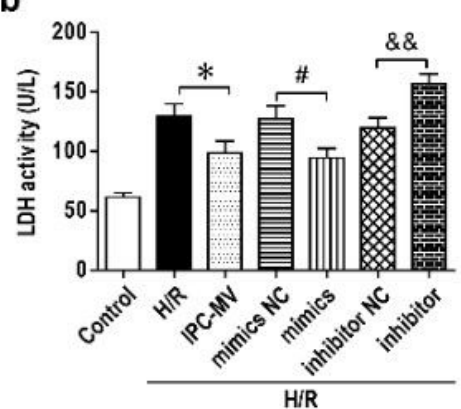

C

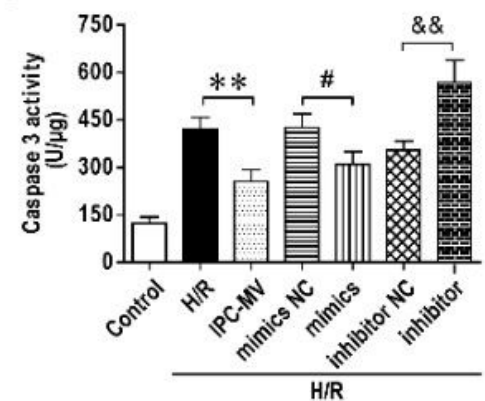

e

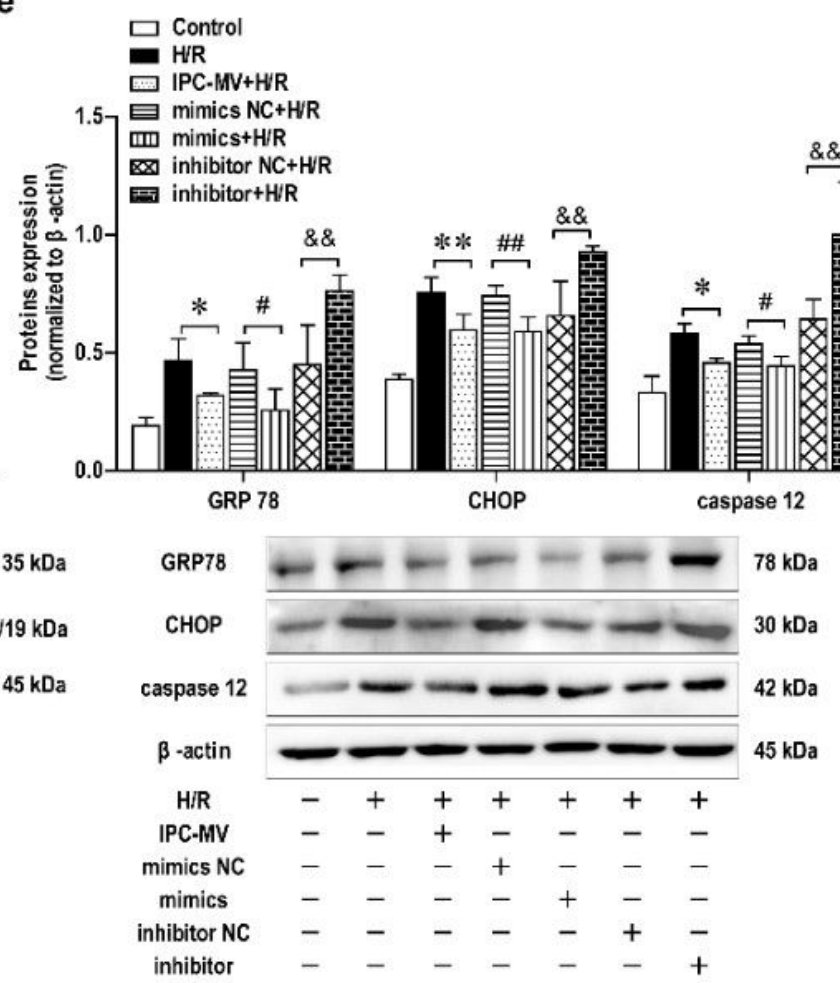

\section{Figure 5}

Protective effects of miR-133a-3p on H9c2 cells against H/R injury. mimics: miR-133a-3p mimics; mimics NC: miR-133a-3p mimics negative control; inhibitor: miR-133a-3p inhibitor; inhibitor NC: miR-133a-3p inhibitor negative control. a-c Effects of miR-133a-3p on cell viability (a), LDH activity (b) and caspase 3 activity (c) in H/R injured H9c2 cells $(n=6)$. d-e Effects of miR-133a-3p on protein expression of caspase 3, cleaved-caspase 3 (d) and, GRP78, CHOP and caspase 12 (e) in H/R injured H9c2 cells detected by Western blot $(n=3) .{ }^{\star} P<0.05,{ }^{*} P<0.01,{ }^{*} * \mathrm{P}<0.001$ vs. $H / R ; \# P<0.05, \# \# P<0.01$ vs. mimics $N C+H / R$; $\& P<0.05, \& \& P<0.01, \& \& \& P<0.001$ vs. inhibitor $N C+H / R$ 
a

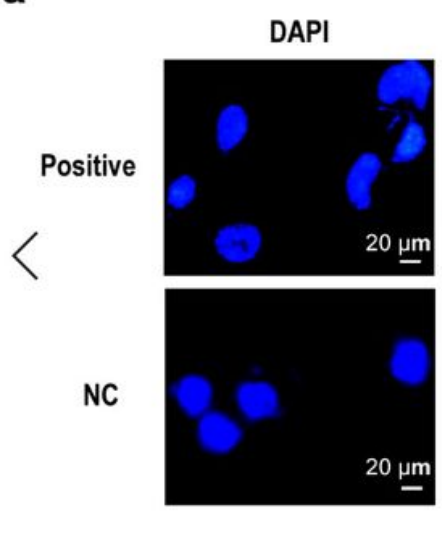

Dil
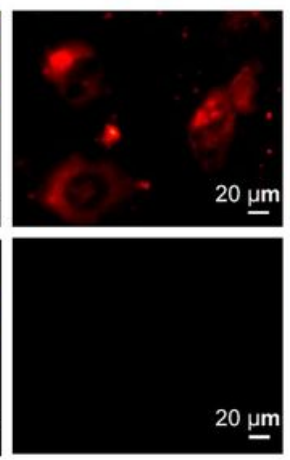

FAM
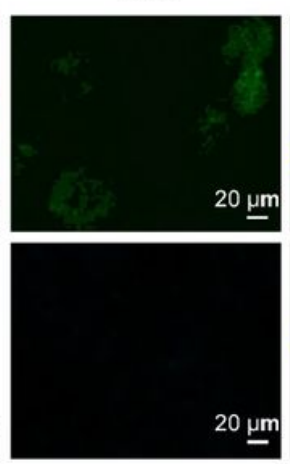

Merge

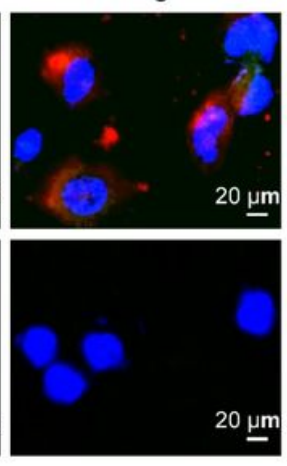

b

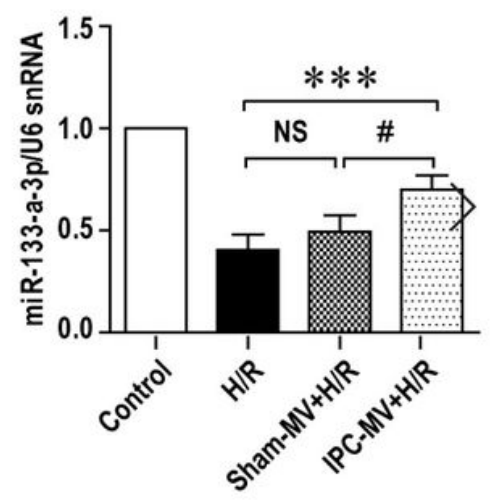

\section{Figure 6}

Transferation of miR-133a-3p to H9c2 cells by HPC-MVs. a Representative fluorescence microscopy images of H9c2 cells co-incubated with HPC-MVs (4 h). green and red labeled HPC-MVs derived from $\mathrm{H} 9 \mathrm{c} 2$ cells that had been transfected with FAM-miR-133a-3p mimics and stained with Dil ( $5 \mu \mathrm{M}), \mathrm{HPC}-$ MVs derived from unlabeled $\mathrm{H} 9 \mathrm{c} 2$ cells were used as negative control (NC). Nuclei were stained with DAPI (blue). Red fluorescent signals indicate HPC-MVs incorporated into $\mathrm{H} 9 \mathrm{c} 2$ cells. Green fluorescent signals indicate miR-133a-3p transferred to H9c2 cells by HPC-MVs. b Effect of IPC-MVs on miR-133a-3p expression in $\mathrm{H} / \mathrm{R}$ injured $\mathrm{H} 9 \mathrm{c} 2$ cells detected by qRT-PCR (normalized to U6 snRNA) $(n=3) .{ }^{\star \star \star} P<0.001$ vs. $H / R ; \# P<0.05$ vs. Sham-MV + H/R; NS means no significant differences 
a

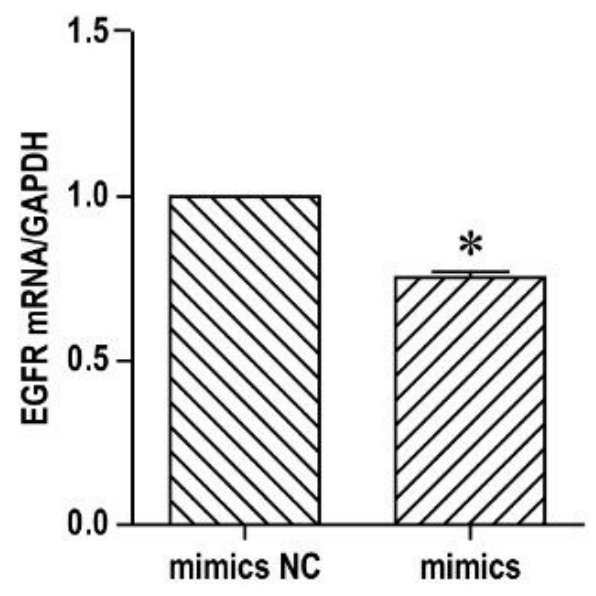

C
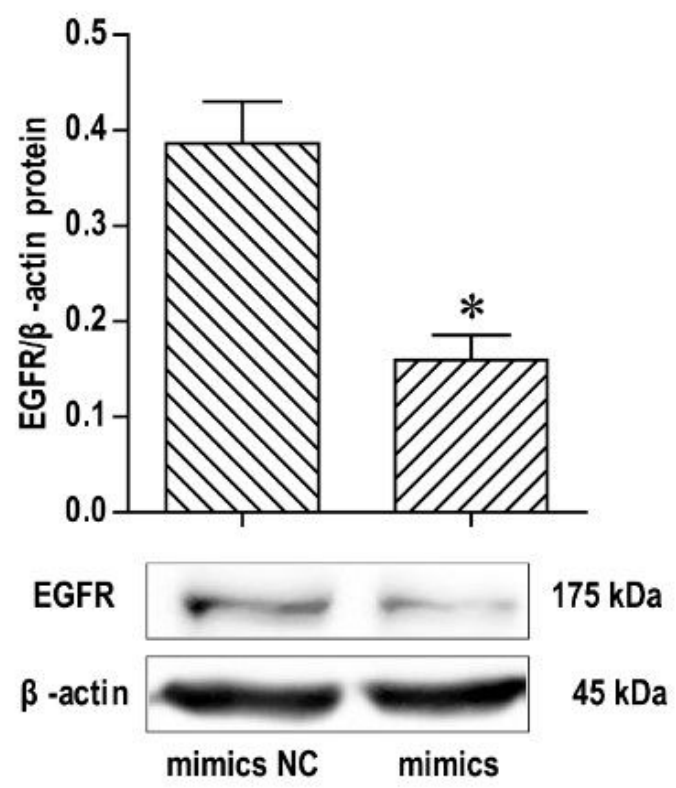

b

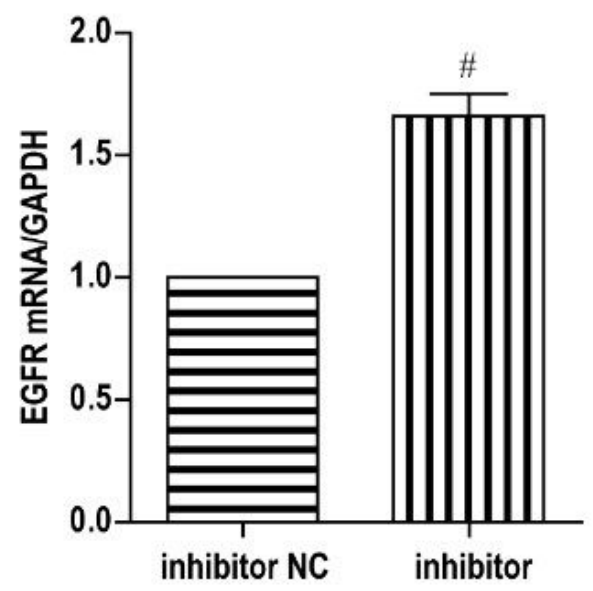

d

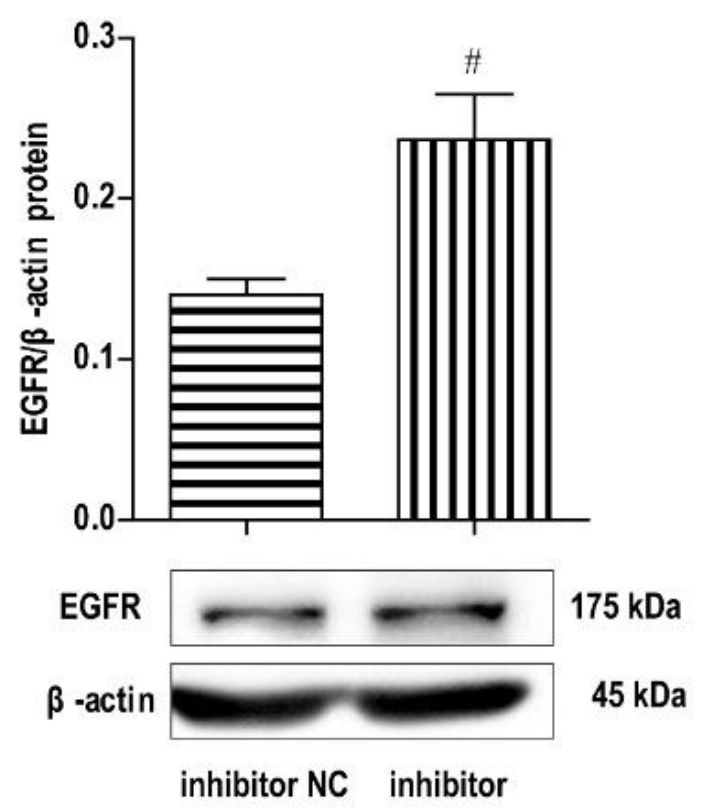

Figure 7

Effects of miR-133a-3p on EGFR mRNA and protein expression in H9c2 cells. a-b Effects of miR-133a-3p mimics (a) and inhibitor (b) on EGFR mRNA expression in H9c2 cells detected by qRT-PCR (normalized to GAPDH). c-d Effects of miR-133a-3p mimics (c) and inhibitor (d) on EGFR protein expression in H9c2 cells detected by Western blot. ${ }^{*}<<0.05$ vs. mimics NC; $\# P<0.05$ vs. inhibitor NC; $n=3$ 
a

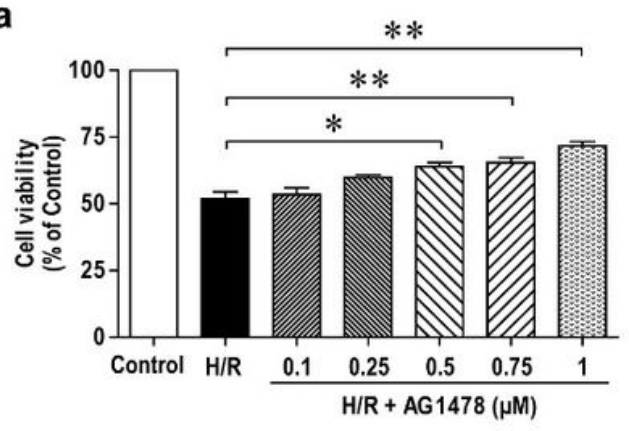

d
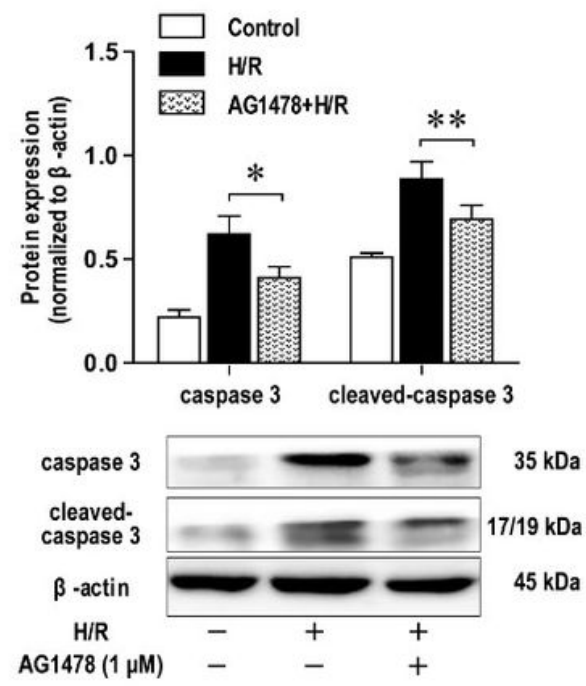

b

C
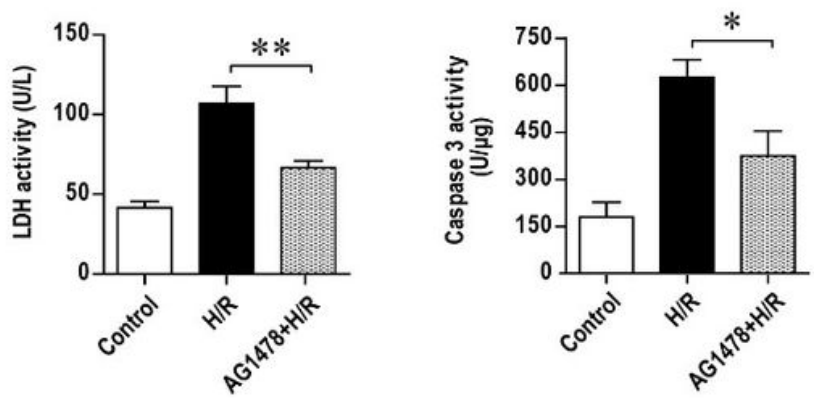

e

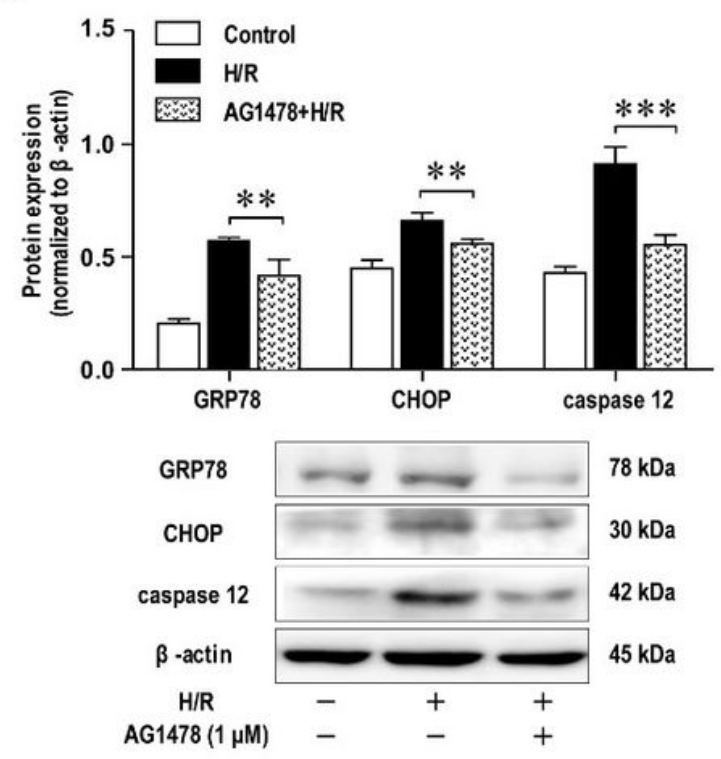

Figure 8

Effects of miR-133a-3p on ERS in H/R injured H9c2 cells. a-c Effects of AG1478 on cell viability (a), LDH activity (b) and caspase 3 activity in H/R injured H9c2 cells (c) ( $n=6)$. d-e Effects of AG1478 (1 $\mu$ M) on protein expression of caspase 3, cleaved-caspase 3 (d) and, GRP78, CHOP and caspase 12 (e) in H/R injured $\mathrm{H} 9 \mathrm{c} 2$ cells detected by Western blot $(n=3)$. ${ }^{\star} P<0.05, * * P<0.01,{ }^{*} * \mathrm{P}<0.001$ vs. H/R

\section{Supplementary Files}

This is a list of supplementary files associated with this preprint. Click to download.

- ARRIVEchecklist.docx 